\title{
Four-Color Flow Cytometric Detection of Retrovirally Expressed Red, Yellow, Green, and Cyan Fluorescent Proteins
}

BioTechniques 30:1028-1034 (May 2001)

\author{
Teresa S. Hawley ${ }^{1}$, William G. \\ Telford $^{2}$, Ali Ramezani ${ }^{1}$, and \\ Robert G. Hawley ${ }^{1,3}$ \\ 1 Jerome H. Holland Laboratory \\ for the Biomedical Sciences, \\ American Red Cross, Rockville, \\ MD, ${ }^{2}$ NCI-NIH, Bethesda, MD, \\ and ${ }^{3}$ George Washington Uni- \\ versity School of Medicine and \\ Health Sciences, Washington, \\ DC, USA
}

\section{INTRODUCTION}

The cloning of the green fluorescent protein (GFP) gene from the luminescent jellyfish Aequorea victoria has led to its widespread use as a marker for gene expression in various biological systems $(5-7,22)$. Site-directed mutations have yielded variants amenable to detection by both fluorescence microscopy and flow cytometry in mammalian cells $(9,11)$. Due to overlapping signals, fluorescence microscopy is used primarily for the detection of individual fluorescent proteins, whereas flow cytometry is employed for the simultaneous analysis of multiple fluorescent proteins $(1-3,17,23,24)$. Using the latter methodology, successful separation of the emission wavelengths of up to three GFP variants has been reported $(4,25)$. In particular, a flow cytometric protocol was recently described in which single-laser excitation at $458 \mathrm{~nm}$ allowed simultaneous analysis of yellow (EYFP), green (EGFP), and cyan (ECFP) emitters in transiently transfected cells (4).

The recent isolation of a red fluorescent protein gene (drFP583) from Discosoma sp., a nonbioluminescent reef coral (Phylum Cnidaria: Class Anthozoa), promises to complement techniques using the Aequorea GFP variants by expanding the range of the spectrum available for detection by more than 50 $\mathrm{nm}$ (19). The subsequent introduction of a human codon-optimized version of the drFP583 gene, DsRed, should further extend the usefulness of this reporter in mammalian systems (Clontech Laboratories, Palo Alto, CA, USA). Visualization of DsRed by fluorescence microscopy in triple-labeling experiments with EYFP and ECFP has been documented by the manufacturer (8); however, a transient transfection assay was used, and the procedure involved fixing the cells and capturing images of the individual fluorescent proteins using separate filter sets. Flow cytometric analysis of DsRed fluorescence in transiently transfected cells was also presented (8). However, it was not known whether the DsRed signal is bright enough to permit detection from stably introduced transgenes in viable mam malian cells using flow cytometric methodology nor whether the spectral properties of this red fluorescent protein are suitable for simultaneous multicolor analyses with GFP variants.

The ability to simultaneously detect four fluorescent proteins in living cells will provide the opportunity to efficiently introduce four (or more) different genes of interest into cell populations using polycistronic retroviral vectors for genetic modification and lineage marking applications $(6,10,15$, $16,20,21)$. To this end, the present study was undertaken to evaluate the feasibility of flow cytometric analysis of DsRed in combination with EYFP, EGFP, and ECFP using dual-laser excitation at 458 and $568 \mathrm{~nm}$. Realizing that not all flow cytometers are equipped with expensive tunable multiline lasers, we also assessed an alternative three-color detection protocol for DsRed, EYFP, and EGFP that is applicable for use on instruments having single-laser excitation at $488 \mathrm{~nm}$.

\section{MATERIALS AND METHODS}

\section{Retroviral Vectors and Cell Line Generation}

The pDsRed1-1, pEYFP-N1, pEGFP-1, and pECFP plasmids encoding DsRed, EYFP, EGFP, and ECFP, respectively, were obtained from Clon- 
tech Laboratories. The MGIN retroviral vector contains the EGFP gene expressed from the murine stem cell virus (MSCV) long terminal repeat on a bicistronic transcript that also contains a downstream neomycin resistance $\left(\mathrm{Neo}^{\mathrm{r}}\right)$ gene under the control of an encephalomyocarditis virus internal ribosome entry site (6). The MRIN, MYIN, and MCIN retroviral vectors containing the DsRed, EYFP, and ECFP genes were similarly constructed by inserting the respective fluorescent protein genes into the same MSCV-based $\mathrm{Neo}^{\mathrm{r}}$ vector backbone $(13,14)$. Stable cell lines expressing the MRIN, MYIN, MGIN, and MCIN retroviral vectors were generated by transduction of GP+E-86 ecotropic packaging cells followed by G418 selection $(400 \mu \mathrm{g} / \mathrm{mL} \quad \mathrm{Ge}$ neticin ${ }^{\circledR}$; Life Technologies, Rockville, MD, USA) and/or cell sorting as previously described (12). GP+E-86 is a derivative of the murine embryonic fibroblast cell line, NIH3T3, and was originally obtained from Dr. Arthur Bank (Columbia University, New York, NY, USA) (18).

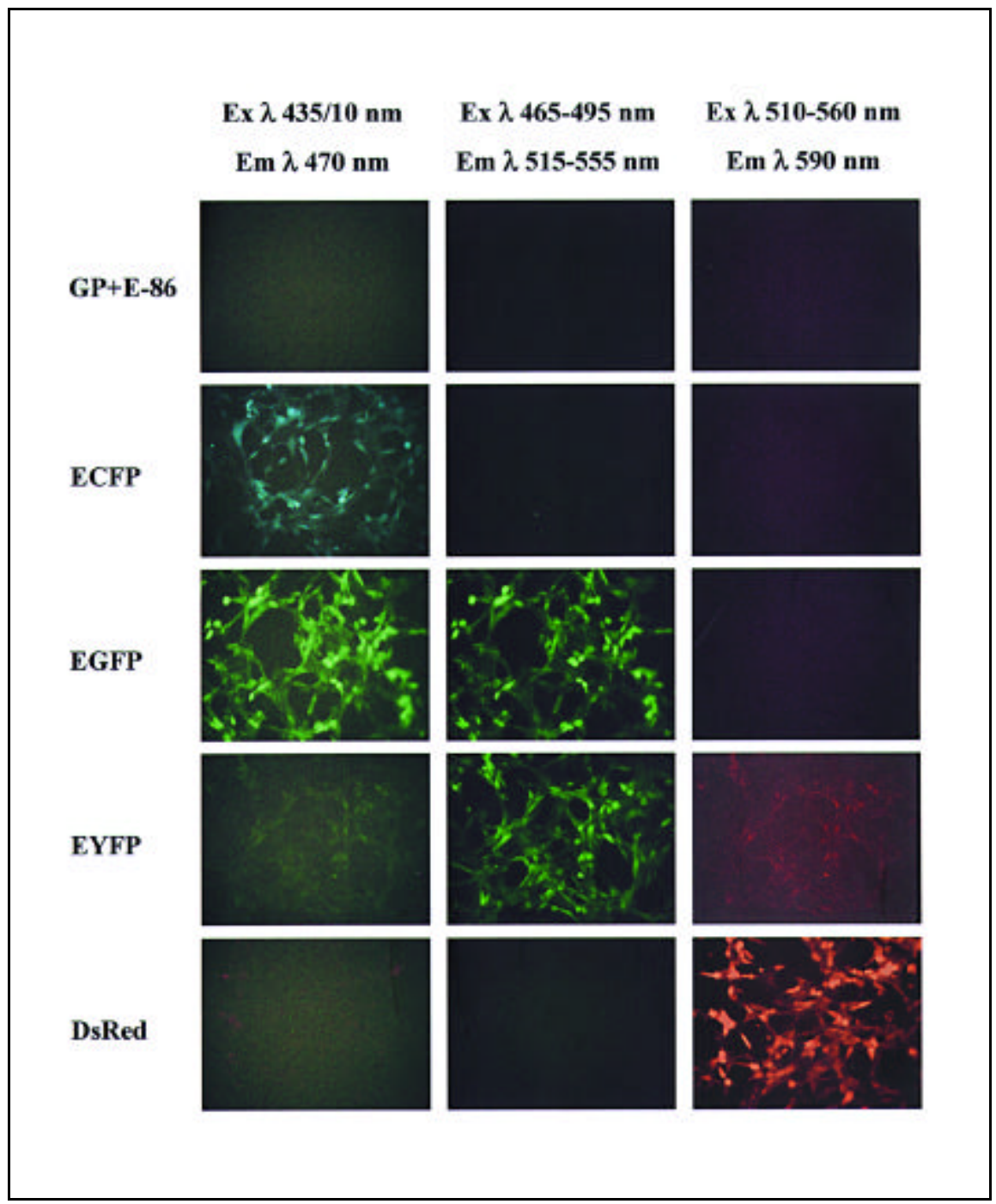

Figure 1. Fluorescence microscopic analysis of four fluorescent proteins using three standard filter sets. GP+E-86 cells individually expressing DsRed, EYFP, EGFP, and ECFP were imaged using each filter set as indicated. The BV-1A filter set (EX 435/10, DM 455, BA 470) used to image the ECFP signal also captured EGFP fluorescence and, to a lesser extent, EYFP fluorescence. The B-2E/C filter set (EX 465-495, DM 505, BA 515-555) used for fluorescein isothiocyanate-like emission could not distinguish between the EGFP and EYFP signals. The G-2A filter set (EX 510-560, DM 575, BA 590) used for phycoerythrin-like emission detected the DsRed signal and the EYFP signal. Images were overexposed to demonstrate spectral overlap.

\section{Fluorescence Microscopy}

Fluorescent proteins were visualized individually under a Nikon Eclipse TE300 inverted fluorescence microscope (Nikon, Melville, NY, USA) equipped with a $100-\mathrm{W}$ mercury arc lamp and a Catseye Digital Camera (Sony Electronics, San Jose, CA, USA). DsRed was detected with a G2 A filter set (EX 510-560, DM 575, BA 590), EYFP, and EGFP with a B-2E/C filter set (EX 465-495, DM 505, BA 515-555), and ECFP with a BV-1A filter set (EX 435/10, DM 455, BA 470).

\section{Flow Cytometry}

Transduced cells were analyzed on a FACSVantage $^{\mathrm{TM}}$ SE (BD Biosciences, San Jose, CA, USA) equipped with Coherent I-90 argon-ion and Coherent I302C krypton-ion lasers (Coherent, Santa Clara, CA, USA). For dual-laser analysis, the primary laser (I-90) was tuned to $458 \mathrm{~nm}$ and the second laser (I-302C) was tuned to $568 \mathrm{~nm}$. The three-beam separation option allowed the krypton-ion laser beam to be directed along the third laser pathway, maximizing spatial separation of the two beams and minimizing both laser noise and signal crosstalk between the signals. For single-laser analysis, the primary laser (I-90) was tuned to $488 \mathrm{~nm}$. Argon-ion 458- and 488-nm laser line power outputs were set at 100 and 70 $\mathrm{mW}$, respectively, and the krypton-ion 568-nm laser line power output was set at $35 \mathrm{~mW}$. Data were acquired using CELLQuest $^{\mathrm{TM}}$ (BD Biosciences). The OmniCompensation option (BD Biosciences) allowed real-time intra- and inter-laser compensations. For comparison purposes, software-based compensation (WinList; Verity Software House, Topsham, ME, USA) was applied to data acquired without hardware compensation in some experiments.

To detect all four fluorescent proteins, EYFP, EGFP, and ECFP were excited at $458 \mathrm{~nm}$, while DsRed was excited at $568 \mathrm{~nm}$. The ECFP signal was split off from the EYFP/EGFP signals using a 500-nm longpass dichroic filter and collected with a 485/22 bandpass (BP) filter. The EYFP and EGFP signals were separated with a 525-nm shortpass dichroic filter and collected 
with a 550/30 BP filter and a 510/20 BP filter, respectively. A 568-nm restriction band filter was placed in front of the 550/30 BP filter to block off stray laser light. The DsRed signal was collected with a 610/30 BP filter with a sharp cutoff at $595 \mathrm{~nm}$.

Three of the four fluorescent proteins-DsRed, EYFP, and EGFPcould be excited at $488 \mathrm{~nm}$. The DsRed signal was split off from the EYFP/ EGFP signals using a 560-nm shortpass dichroic filter and collected with a 585/42 BP filter. The EYFP and EGFP signals were detected as described above. All optical filters were supplied by BD Biosciences or purchased from Omega Optical (Brattleboro, VT, USA) or Chroma Technology (Brattleboro, VT, USA).

To determine whether DsRed could be used in conjunction with surface-labeling fluorochromes, GP+E-86 cells expressing DsRed were incubated with a biotin-conjugated anti-mouse CD90 monoclonal antibody and either streptavidin-PE-Cy5 or streptavidin-PE-Cy7 in a second step. The biotin-conjugated anti-mouse CD90 monoclonal antibody and the streptavidin-PE-Cy5 conjugate were purchased from BD Biosciences. The streptavidin-PE-Cy7 conjugate was purchased from Caltag Laboratories (Burlingame, CA, USA). The antibody and secondary reagents were used at concentrations recommended by the manufacturers.

\section{RESULTS AND DISCUSSION}

\section{Spectral Overlap of Fluorescent Proteins Illustrated by Fluorescence Microscopy}

Permanent cell lines stably expressing DsRed, EYFP, EGFP, ECFP, and DsRed plus EGFP were generated by retroviral-mediated gene transfer (see Materials and Methods). No toxicity due to the sustained expression of DsRed or any of the GFP variants was observed. Before flow cytometric analysis, cells expressing individual fluorescent protein genes were evaluated by fluorescence microscopy using standard filter sets that closely matched the excitation and emission maxima of the respective fluorescent proteins (Table 1). All four fluorescent proteins were easily

Table 1. Spectral Characteristics of the Red, Yellow, Green, and Cyan Fluorescent Proteins ${ }^{\text {a }}$

\begin{tabular}{|llcc|}
\hline Protein & Color & $\begin{array}{c}\text { Excitation } \\
\text { Maximum }(\mathbf{n m})\end{array}$ & $\begin{array}{c}\text { Emission } \\
\text { Maximum (nm) }\end{array}$ \\
\hline DsRed & red & 558 & 583 \\
EYFP & yellow & 514 & 527 \\
EGFP & green & 489 & 508 \\
ECFP & cyan & 434 & 477 \\
aData from Reference 8. & & \\
\hline
\end{tabular}

Table 2. Instrument Settings for Analysis of Four Fluorescent Proteins Expressed by GP+E-86 Cells Using Dual-Laser (458 and 568 nm) Flow Cytometry ${ }^{\mathrm{a}}$

\begin{tabular}{|c|c|c|c|c|c|c|}
\hline \multirow{2}{*}{ Parameter } & \multirow{2}{*}{$\begin{array}{c}\text { Fluorescent } \\
\text { Protein }\end{array}$} & \multirow{2}{*}{$\begin{array}{c}\text { PMT } \\
\text { Voltage }\end{array}$} & \multicolumn{4}{|c|}{ Compensation } \\
\hline & & & (-\%FL1) & (-\%FL2) & (-\%FL3) & (-\%FL4) \\
\hline FL1 & EGFP & 438 & - & $67.6 \%$ & $61.3 \%$ & $0 \%$ \\
\hline FL2 & EYFP & 753 & $49.2 \%$ & - & $6 \%$ & $0 \%$ \\
\hline FL3 & ECFP & 380 & $18.3 \%$ & $0 \%$ & - & $0 \%$ \\
\hline FL4 & DsRed & 483 & $0 \%$ & $0 \%$ & $0 \%$ & - \\
\hline \multicolumn{7}{|c|}{$\begin{array}{l}\text { aEach voltage setting was adjusted with non-transduced negative control cells } \\
\text { and cells expressing a particular fluorescent protein to position the fluorescence } \\
\text { signal on scale. Compensation was set using CELLQuest and the } \\
\text { OmniCompensation circuitry. Parameter settings were fine-tuned to achieve a } \\
\text { balance of compensations. }\end{array}$} \\
\hline
\end{tabular}

visualized, but spectral overlap was prominent, especially among the GFP variants (Figure 1). Considerable fluorescence from EGFP and, to a lesser extent, EYFP was observed when the cells were viewed under the filter set used for ECFP detection. In addition, EYFP and EGFP signals were both detected with the filter set designed for fluorescein isothiocyanate fluorescence. As expected from their spectral characteristics, some of the EYFP signal was detected with the filter set used for DsRed fluorescence, whereas no signals were detected from EGFP and ECFP.

\section{Flow Cytometric Detection of DsRed and Three GFP Variants}

In flow cytometry, the problem of spectral overlap can be dealt with by employing the appropriate optical filter configuration and real-time electronic (hardware) or post-analysis softwarebased compensation $(1,2,4,17,24,25)$. Because simultaneous flow cytometric analysis of the three GFP variants under study was recently demonstrated using single-laser excitation at $458 \mathrm{~nm}$ (4), we reasoned that the resolution of fluorescence signals from all four fluorescent proteins might be attainable by exciting EYFP, EGFP, and ECFP at $458 \mathrm{~nm}$ and DsRed at a wavelength close to its excitation maximum (558 $\mathrm{nm})$. Accordingly, cells expressing individual fluorescent proteins were analyzed on the flow cytometer using dual-laser excitation at 458 and 568 $\mathrm{nm}$. This latter excitation wavelength was chosen because it is available on the tunable multiline krypton-ion laser. At $458 \mathrm{~nm}$, all four fluorescent proteins are excited, approximately equally well for EGFP and ECFP (60\% of maximum absorbance), less well for DsRed $(<20 \%$ of maximum absorbance), and the least well for EYFP ( $<10 \%$ of maximum absorbance) (8). The resolution of EYFP, EGFP, and ECFP fluorescence was achieved by intra-laser compensation (Table 2). Po- 
Table 3. Instrument Settings for Analysis of Three Fluorescent Proteins Expressed by GP+E-86 Cells Using Single-Laser (488 nm) Flow Cytometrya

\begin{tabular}{|lccccc|}
\hline Parameter & $\begin{array}{l}\text { Fluorescent } \\
\text { Protein }\end{array}$ & $\begin{array}{c}\text { PMT } \\
\text { Voltage }\end{array}$ & (-\%FL1) & $\begin{array}{c}\text { Compensation } \\
\text { (-\%FL2) }\end{array}$ & (-\%FL3) \\
\hline FL1 & EGFP & 457 & - & $52.8 \%$ & $0 \%$ \\
FL2 & EYFP & 619 & $78.8 \%$ & - & $1.6 \%$ \\
FL3 & DsRed & 381 & $0 \%$ & $67.2 \%$ & - \\
aParameter settings were adjusted as described in Table 2 for the dual-laser \\
analysis.
\end{tabular}

tential interference of these signals with DsRed detection could have been dealt with by inter-laser compensation. However, the latter process was obviated by selective use of a 610/30 BP filter, which excluded signals from these three fluorescent proteins from the DsRed fluorescence channel. Conversely, because of its red-shifted emission spectrum, DsRed fluorescence generated from either 458- or 568-nm excitation did not interfere with the signals of the other three fluorescent proteins. At 568-nm excitation, no signals were elicited from EYFP, EGFP, or ECFP. Under these conditions, the signals from all four fluorescent proteins were successfully resolved in real time by careful pair-wise compensations between detectors (Figure 2).

\section{Three-Color Fluorescent Protein Detection Using Single-Laser (488-nm) Excitation}

DsRed has a minor excitation peak around $488 \mathrm{~nm}$ and can be excited reasonably efficiently at this wavelength (40\% of maximum absorbance) (8). Since many flow cytometers are

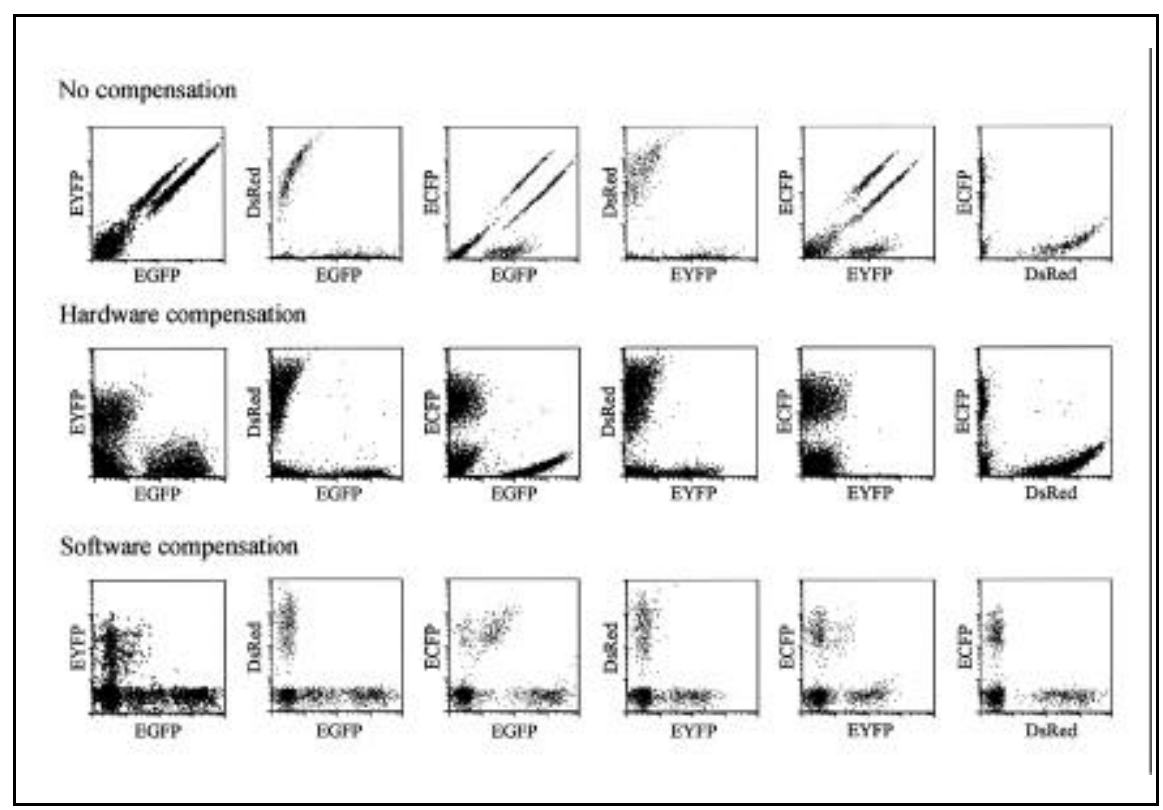

Figure 2. Flow cytometric analysis of four fluorescent proteins using excitation wavelengths at 458 and $568 \mathrm{~nm}$. Resolution of fluorescence signals from DsRed, EYFP, EGFP, and ECFP was obtained in a mixture of GP+E-86 cells expressing the individual proteins (together with non-transduced negative control cells). Top row: uncompensated data. Middle row: data generated in real time with electronic (hardware) compensation (CELLQuest and OmniCompensation; see Table 2 for instrument settings). Note that the compensation required to completely eliminate spectral overlap of intense fluorescence signals resulted in an apparent overcompensation of cells expressing lower levels of the fluorescent proteins (see Reference 4). Bottom row: the results obtained by applying post-analysis software compensation (WinList) to the uncompensated data are presented for comparison purposes. 
equipped with an argon-ion laser that can be tuned to $488 \mathrm{~nm}$, the possibility of analyzing the DsRed/EYFP/EGFP combination using single-laser excitation at this wavelength was evaluated. As shown in Figure 3, fluorescence signals from the three fluorescent proteins were readily detected and could be distinguished in real time under the conditions employed (Table 3 ).

\section{Simultaneous Analysis of DsRed in Conjunction with EGFP, PE-Cy5, or PE-Cy7}

Simultaneous detection and discrimination of DsRed and EGFP fluorescence using dual-laser excitation was

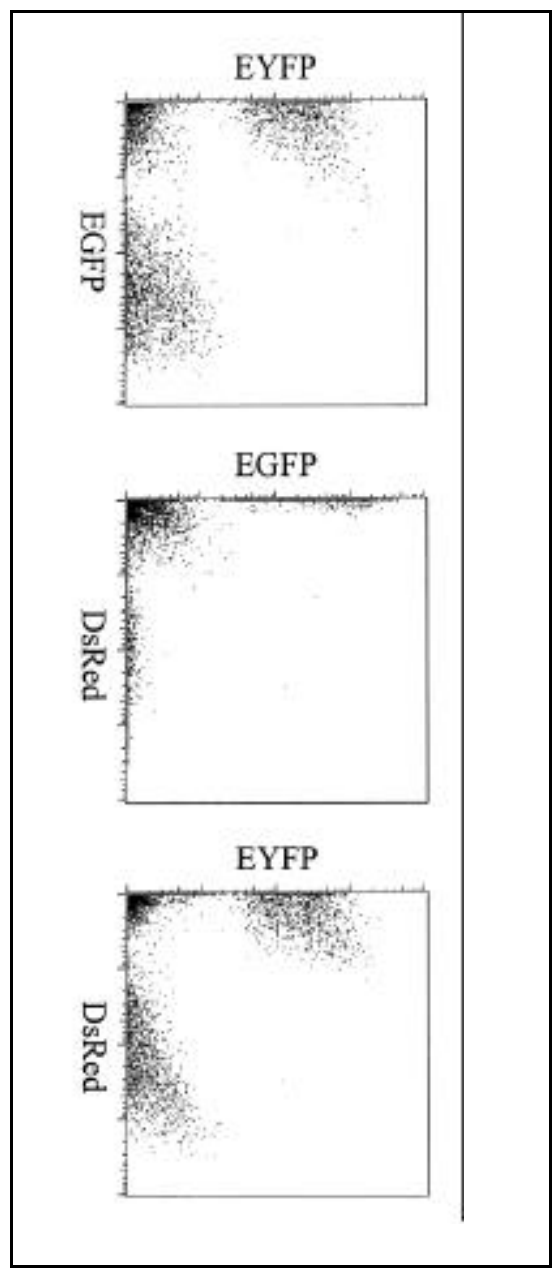

Figure 3. Flow cytometric analysis of three fluorescent proteins using single-laser excitation at $488 \mathrm{~nm}$. Real-time resolution of fluorescence signals from DsRed, EYFP, and EGFP in a mixture of GP+E-86 cells expressing the individual proteins (together with non-transduced negative control cells). See Table 3 for instrument settings. demonstrated using populations of cells expressing either or both of these fluorescent proteins (Figure 4A). Cells simultaneously expressing DsRed and EGFP could also be easily discriminated from cells expressing the individual fluorescent proteins using the singlelaser excitation procedure (Figure 4B). Based on these latter results, we predicted that, for cytometers equipped with four detectors off of a primary 488-nm laser beam, cells marked with the DsRed/EYFP/EGFP combination could also be labeled with fluorochromes emitting beyond 600 or 650 $\mathrm{nm}$, depending on whether a 585/42 BP filter or a $610 / 30$ BP filter was used for DsRed detection. Possible candidates included PE-Texas Red, PE-Cy5, PerCP-Cy5.5, and PE-Cy7. Analysis of DsRed-expressing GP+E-86 cells labeled with anti-CD90-PE-Cy5 or antiCD90-PE-Cy7 confirmed the ability to simultaneously detect DsRed and a surface antigen with these monoclonal antibody-fluorochrome conjugates using this protocol (Figure 4, C-F).

\section{CONCLUDING REMARKS}

We report the development of two flow cytometry methods to detect the newly described DsRed and up to three GFP variants. In one method, we adapted an optical filter configuration that was recently introduced to simultaneously detect EYFP, EGFP, and ECFP and employed dual-laser line excitation at 458 and $568 \mathrm{~nm}$ to demonstrate the feasibility of identifying distinct cell populations stably marked with these three GFP variants plus DsRed. Flow cytometric data on simultaneous detection of DsRed and EGFP at the singlecell level was also presented. Simultaneous detection of the EYFP/EGFP, EYFP/ECFP, or EGFP/ECFP combinations in individual cells was previously shown using 458-nm excitation (4). More recently, we have generated permanent cell lines expressing DsRed plus EYFP, EGFP, and ECFP (unpublished data). Collectively, these observations establish that it is possible with the dual-laser technique described here

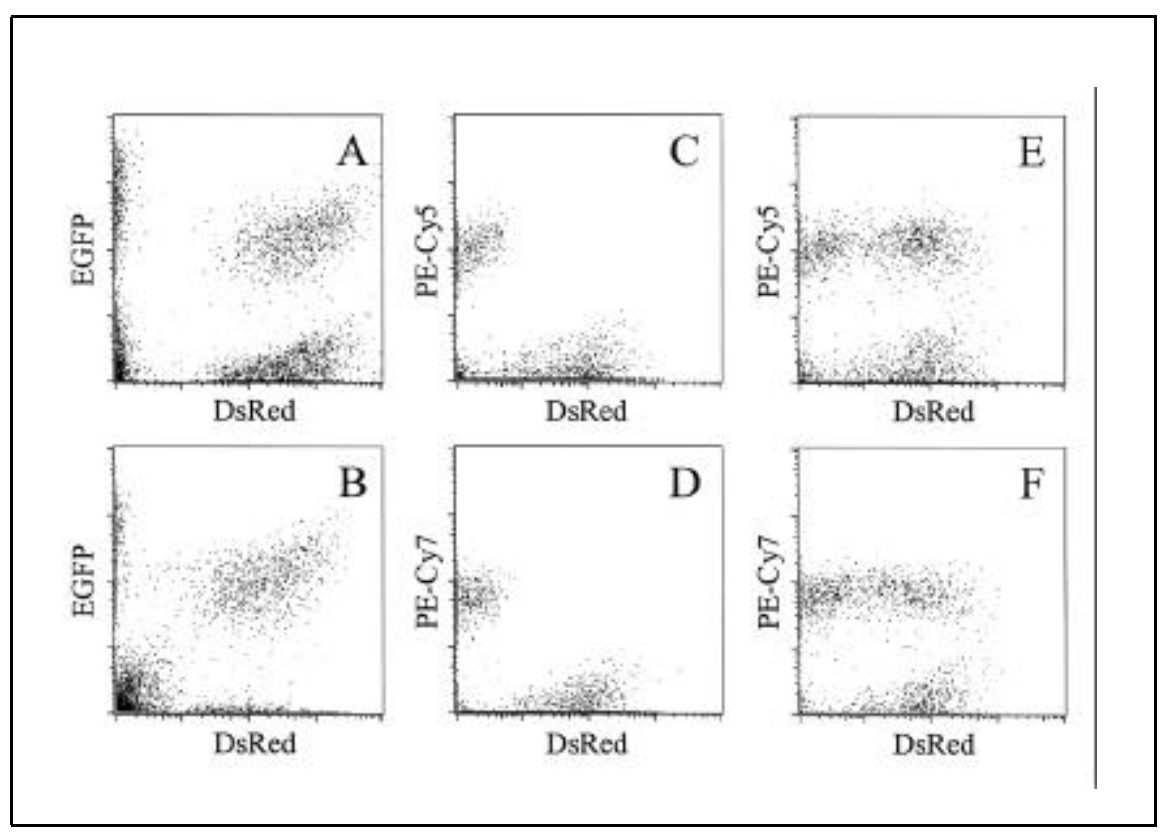

Figure 4. Simultaneous analysis of DsRed in conjunction with EGFP or a surface antigen. Plot A shows simultaneous detection of DsRed and EGFP in a mixture of GP+E-86 cells expressing either or both fluorescent proteins (together with non-transduced negative control cells) using dual-laser (458 and $568 \mathrm{~nm}$ ) excitation. Plot B shows similar detection using single-laser $(488 \mathrm{~nm})$ excitation. Plots C and D indicate complete resolution of DsRed fluorescence and a surface-labeling fluorochrome (PE-Cy5 or PE-Cy7) using single-laser (488-nm) excitation. A $610 \mathrm{~nm}$ shortpass dichroic filter split the signals detected with the 585/42 BP filter for DsRed and the signals detected with the 675/20 BP filter for PE-Cy5. For PE-Cy7, a $745 \mathrm{~nm}$ EFLP filter was used. Plot E shows simultaneous detection of DsRed and PECy5 in a mixture of singly and doubly labeled GP+E-86 cells. Plot F shows simultaneous detection of DsRed and PE-Cy7 in a mixture of singly and doubly labeled GP+E-86 cells. 
to directly detect and viably sort subpopulations of cells simultaneously expressing DsRed and various combinations of these three GFP variants for mammalian studies of gene function and cell tracking $(6,10,15,16,20,21)$.

We also introduced a new three-color detection scheme involving DsRed, EYFP, and EGFP that uses single-laser excitation at $488 \mathrm{~nm}$ and provided flow cytometric data showing simultaneous detection of DsRed and EGFP, DsRed and PE-Cy5, or DsRed and PE-Cy7 at the single-cell level. It is anticipated that this DsRed-based protocol will have broader utility in triple-labeling experiments with fluorescent proteins than three-color flow cytometric methods that include ECFP or the blue-shifted variant of GFP because the latter require excitation with laser lines that are not available on standard flow cytometers $(4,25)$.

\section{ACKNOWLEDGMENTS}

T.S.H. and W.G.T. contributed equally to this work. T.S.H. gratefully ac- knowledges John Swanson (BD Biosciences) for invaluable instrumentation support.

\section{REFERENCES}

1.Anderson, M.T., N. Baumgarth, R.P. Haugland, R.M. Gerstein, T. Tjioe, and L.A. Herzenberg. 1998. Pairs of violet-light-excited fluorochromes for flow cytometric analysis. Cytometry 33:435-444.

2.Anderson, M.T., I.M. Tjioe, M.C. Lorincz, D.R. Parks, L.A. Herzenberg, G.P. Nolan, and L.A. Herzenberg. 1996. Simultaneous fluorescence-activated cell sorter analysis of two distinct transcriptional elements within a single cell using engineered green fluorescent proteins. Proc. Natl. Acad. Sci. USA 93:85088511.

3.Baumann, C.T., C.S. Lim, and G.L. Hager. 1998. Simultaneous visualization of the yellow and green forms of the green fluorescent protein in living cells. J. Histochem. Cytochem. 46:1073-1076.

4.Beavis, A.J. and R.F. Kalejta. 1999. Simultaneous analysis of the cyan, yellow and green fluorescent proteins by flow cytometry using single-laser excitation at $458 \mathrm{~nm}$. Cytometry 37:68-73.

5.Chalfie, M., Y. Tu, G. Euskirchen, W.W. Ward, and D.C. Prasher. 1994. Green fluorescent protein as a marker for gene expression. Science 263:802-805.

6.Cheng, L., C. Du, D. Murray, X. Tong, Y.A. Zhang, B.P. Chen, and R.G. Hawley. 1997. A GFP reporter system to assess gene transfer and expression in viable human hematopoietic progenitors. Gene Ther. 4:1013-1022.

7.Cheng, L., J. Fu, A. Tsukamoto, and R.G. Hawley. 1996. Use of green fluorescent protein (GFP) variants to monitor gene transfer and expression in mammalian cells. Nat. Biotechnol. 14:606-609.

8.Clontech Laboratories. 1999. Living Colors $^{\mathrm{TM}}$ red fluorescent protein. CLON TECHniques XIV:2-6. (http://www.clontech. com/archive/OCT99UPD/RFP.html)

9.Cormack, B.P., R.H. Valdivia, and S. Falkow. 1996. FACS-optimized mutants of the green fluorescent protein (GFP). Gene 173:33-38.

10.De Sepulveda, P., K. Okkenhaug, J.L. Rose, R.G. Hawley, P. Dubreuil, and R. Rottapel. 1999. Socs1 binds to multiple signalling proteins and suppresses Steel factor-dependent proliferation. EMBO J. 18:904-915.

11.Haseloff, J. 1999. GFP variants for multispectral imaging of living cells. Methods Cell. Biol. 58:139-151.

12.Hawley, R.G., T.S. Hawley, A.Z.C. Fong, C. Quinto, M. Collins, J.P. Leonard, and S.J. Goldman. 1996. Thrombopoietic potential and serial repopulating ability of murine hematopoietic stem cells constitutively expressing interleukin-11. Proc. Natl. Acad. Sci. USA 93:10297-10302.

13.Hawley, R.G., F.H.L. Lieu, A.Z.C. Fong, S.J. Goldman, J.P. Leonard, and T.S. Hawley. 1996. Retroviral vectors for production of interleukin-12 in the bone marrow to induce a graft-versus-leukemia effect. Ann. N.Y. Acad.
Sci. 795:341-345.

14.Hawley, R.G., F.H.L. Lieu, A.Z.C. Fong, and T.S. Hawley. 1994. Versatile retroviral vectors for potential use in gene therapy. Gene Ther. 1:136-138.

15.Huppa, J.B. and H.L. Ploegh. 1997. In vitro translation and assembly of a complete $\mathrm{T}$ cell receptor-CD3 complex. J. Exp. Med. 186:393403.

16.Leung, B.L., L. Haughn, A. Veillette, R.G. Hawley, R. Rottapel, and M. Julius. 1999. $\mathrm{Tc} \operatorname{R} \alpha \beta$ independent CD28 signaling and costimulation require non-CD4-associated Lck. J. Immunol. 163:1334-1341.

17.Lybarger, L., D. Dempsey, G.H. Patterson, D.W. Piston, S.R. Kain, and R. Chervenak. 1998. Dual-color flow cytometric detection of fluorescent proteins using single-laser (488$\mathrm{nm}$ ) excitation. Cytometry 31:147-152 .

18.Markowitz, D., S. Goff, and A. Bank. 1988. A safe packaging line for gene transfer: separating viral genes on two different plasmids. J. Virol. 62:1120-1124.

19.Matz, M.V., A.F. Fradkov, Y.A. Labas, A.P. Savitsky, A.G. Zaraisky, M.L. Markelov, and S.A. Lukyanov. 1999. Fluorescent proteins from nonbioluminescent Anthozoa species. Nat. Biotechnol. 17:969-973.

20.Persons, D.A., J.A. Allay, J.M. Riberdy, R.P. Wersto, R.E. Donahue, B.P. Sorrentino, and A.W. Nienhuis. 1998. Use of the green fluorescent protein as a marker to identify and track genetically modified hematopoietic cells. Nat. Med. 4:1201-1205.

21.Roy, S.N., R. Procyk, B.J. Kudryk, and C.M. Redman. 1991. Assembly and secretion of recombinant human fibrinogen. J. Biol. Chem. 266:4758-4763.

22.Tsien, R.Y. 1998. The green fluorescent protein. Annu. Rev. Biochem. 67:509-544.

23. Yang, T.-T., S.R. Kain, P. Kitts, A. Kondepudi, M.M. Yang, and D.C. Youvan. 1996. Dual color microscopic imagery of cells expressing the green fluorescent protein and a red-shifted variant. Gene 173:19-23.

24. Yang, T.-T., P. Sinai, G. Green, P.A. Kitts, Y.T. Chen, L. Lybarger, R. Chervenak, G.H. Patterson, D.W. Piston, and S.R. Kain. 1998. Improved fluorescence and dual color detection with enhanced blue and green variants of the green fluorescent protein. J. Biol. Chem. 273:8212-8216.

25.Zhu, J., M.L. Musco, and M.J. Grace. 1999. Three-color flow cytometry analysis of tricistronic expression of eBFP, eGFP, and eYFP using EMCV-IRES linkages. Cytometry 37:5159 .

Received 5 September 2000; accepted 7 November 2000.

Address correspondence to:

Dr. Robert G. Hawley

Hematopoiesis Department

Holland Laboratory

American Red Cross

15601 Crabbs Branch Way

Rockville, MD 20855, USA

e-mail: hawleyr@usa.redcross.org 